EDITORIAL

\title{
Distinguishing hypertrophic cardiomyopathy from athlete's heart: a clinical problem of increasing magnitude and significance
}

\section{B J Maron}

Heart 2005;91:1380-1382. doi: 10.1136/hrt.2005.060962

\begin{abstract}
As hypertrophic cardiomyopathy is a common cause of sudden deaths among athletes, differentiating this condition from the non-pathological "athlete's heart" presents an important challenge
\end{abstract}

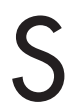
udden unexpected deaths of young trained athletes are highly visible events with substantial impact on the physician and lay communities. ${ }^{1}$ Although a focal point first in the USA in the early $1980 \mathrm{~s}^{2}$ this issue has now become one of increasing concern in many parts of the industrialised world (particularly Europe). ${ }^{3}$ The visibility accorded this medical issue is underscored by the highly publicised sudden deaths of several elite professional athletes, including Hank Gathers and Reggie Lewis in the USA, and more recently several athletes from Europe, South America, and Africa including Marc-Vivian Foe (Cameroon; soccer) who was known to have hypertrophic cardiomyopathy (HCM). ${ }^{5}$

\section{IMPLICATIONS OF DIFFERENTIATING HEART DISEASE FROM ATHLETE'S HEART} Interest in athletic field deaths has also accelerated because of the recognition that these catastrophic events are probably more common than previously thought, occur in young people of both sexes and under-served minorities, and that the responsible but usually unsuspected structural cardiovascular diseases are often clinically identifiable-including by pre-participation screening. ${ }^{4}$ Furthermore, application of the implantable cardioverter-defibrillator to selected high risk patients has made diagnosis of these diseases, and disqualification from certain sports, ${ }^{16-8}$ particularly relevant.

The cardiac diseases that predispose athletes to lethal ventricular tachyarrhythmias have been documented in several necropsy based studies. ${ }^{13}{ }^{10}$ In the USA, the single most common cause of athletic field deaths is HCM, accounting for about one third. ${ }^{139}$ Consequently, differential diagnosis between HCM and physiologic non-pathologic left ventricular (LV) hypertrophy associated with systematic training (commonly referred to as "athlete's heart") has become a not uncommon clinical dilemma. ${ }^{11}$ This diagnostic distinction has important implications since identification of cardiovascular diseases associated with sudden death may be the basis for disqualification from competition to minimise
Minneapolis, MN 55407 org risk. ${ }^{8}$ Conversely, improper over-diagnosis of cardiac disease may lead to unnecessary withdrawal from athletics, thereby depriving that individual of the varied psychological and sometimes economic benefits of competitive sports. Interest in application of non-invasive markers that may resolve such differential diagnosis has increased with the greater visibility of athletic field deaths ${ }^{1-35}$ and focus on pre-participation screening in both the USA and Europe. ${ }^{35}$ Furthermore, legal liability incurred by evaluating competitive athletes with cardiovascular disease (presently most substantial in the USA) has raised the general level of anxiety surrounding these clinical situations.

\section{STRATEGIES FOR DIFFERENTIAL DIAGNOSIS}

An algorithm for differential diagnosis between HCM (with mild phenotypic expression) and extreme physiologic hypertrophy of athlete's heart has evolved, with particular relevance to trained athletes in whom left ventricular (LV) wall thickness falls into a "grey zone" of overlap between these clinical scenarios (fig 1) - that is, ventricular septal thickness of 13-15 mm present in $2 \%$ of highly trained male athletes. ${ }^{11}$ Of note, this clinical distinction is relevant only to nonobstructive HCM under resting (basal) conditions, in which mitral valve systolic anterior motion (SAM) is absent, since SAM is inconsistent with athlete's heart and strongly suggests HCM.

\section{Genetic testing}

The most definitive resolution of this important differential diagnosis can come from genetic testing. Indeed, a rapid genetic test is now available, ${ }^{12}$ analysing by direct DNA sequencing mutations in the eight most common HCM causing genes. While a positive test result in an athlete can resolve the diagnostic ambiguity between athlete's heart and HCM, there is however significant potential for false negative test results in which a HCM diagnosis cannot be excluded.

\section{Cavity dimension}

In lieu of genetic testing, other echocardiographic parameters may be useful to distinguish between athlete's heart and HCM, including LV end diastolic cavity enlargement $>55 \mathrm{~mm}$. LV

Abbreviations: HCM, hypertrophic cardiomyopathy; LV,
left ventricular; SAM, systolic anterior motion 


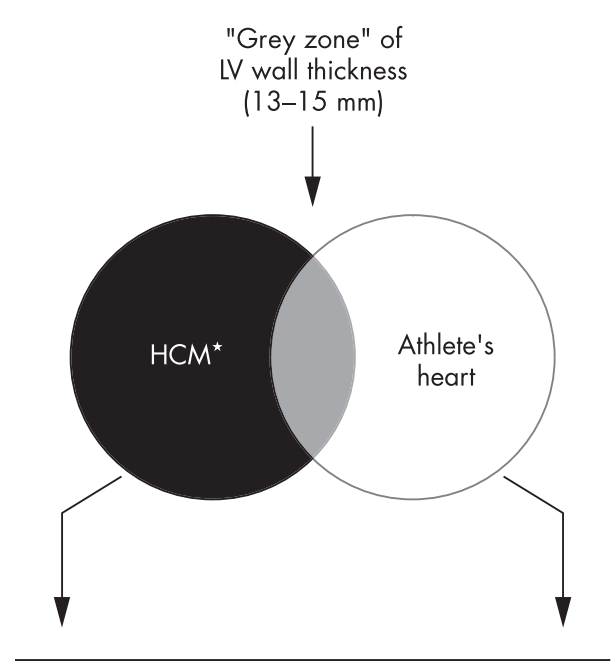

$+\quad$ Unusual patterns of $\mathrm{LVH}$
$+\quad \mathrm{LV}$ cavity $<45 \mathrm{~mm}$
$-\quad \mathrm{LV}$ cavity $>55 \mathrm{~mm}$
$+\quad$ Marked LA enlargement
$+\quad$ Bizarre ECG patterns
$+\quad$ Abnormal LV filling
$+\quad$ Female sex
$-\quad \downarrow$ Thickness with deconditioning
$+\quad$ Family history of $\mathrm{HCM}$
$-\quad$ Max $\mathrm{VO}_{2}>45 \mathrm{ml} / \mathrm{kg} / \mathrm{min}$
$>110 \% \mathrm{predicted}$

Figure 1 Criteria used to distinguish hypertrophic cardiomyopathy (HCM) from athlete's heart when the left ventricular (LV) wall thickness is within the shaded "grey zone" of overlap, consistent with both diagnoses. $\downarrow$ indicates decreased; LA, left atrial; LVH, left ventricular hypertrophy. Reproduced from Maron et al, "1 with permission of American Heart Association.

dimensions in this range are common in trained athletes, but rare in HCM in which cavity size is often $<45 \mathrm{~mm}$ and dimensions of $>55 \mathrm{~mm}$ usually occur only in the end stage phase with progressive heart failure and systolic dysfunction. ${ }^{1}$ Therefore, in some instances, LV cavity dimension is useful in distinguishing athlete's heart from HCM.

\section{LV filling}

Most HCM patients show abnormal pulsed or tissue Doppler diastolic indexes of LV filling, independent of whether heart failure or outflow obstruction are present. ${ }^{1}$ In contrast, trained athletes invariably demonstrate normal LV filling patterns. ${ }^{1}$ Consequently, in a trained athlete suspected of having HCM, a distinctly abnormal Doppler filling pattern supports this diagnosis, while a normal study is consistent with either HCM or athlete's heart.

\section{Sex}

Sex differences in cardiac dimensions are often relevant to differential diagnosis. Highly trained female athletes rarely show absolute LV wall thicknesses $>11 \mathrm{~mm} .{ }^{1}$ Indeed, of 600 elite women athletes, none had LV thickness in the range compatible with the diagnosis of HCM $(\geqslant 13 \mathrm{~mm}){ }^{13}$ Therefore, female athletes presenting with "grey zone" LV wall thickness of 13-15 mm (and normal cavity size) are most likely to have HCM.

\section{Deconditioning}

Forced detraining may be a useful manoeuvre to resolve differential diagnosis between athlete's heart and HCM. Elite athletes with LV hypertrophy may show reduction in wall thickness (of about 2-5 mm) over short deconditioning periods (about three months). ${ }^{1}$ However, identification of such changes in wall thickness with deconditioning requires: (1) substantial compliance to interrupt the training of highly motivated athletes; and (2) serial echocardiographic or magnetic resonance imaging studies of technical quality suitable for comparison. Decreased LV wall thickness associated with deconditioning substantiates that LV hypertrophy is a physiologic consequence of athletic training, as such changes in wall thickness are inconsistent with pathologic hypertrophy of HCM. ${ }^{14}$

\section{Type of sport training}

The specific nature of athletic training has a major influence on LV dimension. For example, rowing sports and cycling are most commonly associated with LV wall thickness $\geqslant 13 \mathrm{~mm}$. Conversely, isometric (or power) sports, such as weightlifting or wrestling, are not associated with absolute increases in LV wall thickness $>12 \mathrm{~mm} .{ }^{15}$ Therefore, in assessing whether an athlete with LV hypertrophy has HCM, detailed knowledge of the training regimen is important.

In this issue of Heart, Anastasakis et al ${ }^{16}$ use cardiopulmonary exercise testing to differentiate between HCM patients and elite male athletes. These authors specifically offer novel observations regarding endurance compared to strength training in athletes without structural heart disease. Cardiopulmonary exercise parameters, such as peak oxygen consumption, reliably distinguished HCM from endurance athletes (middle/long distance runners), but not from strength trained athletes (weightlifters). This observation adds an important and much needed piece of information to this area of investigation, contributing a measure of clarity to clinical strategies for differentiating pathologic from physiologic hypertrophy.

\section{CONCLUSIONS}

In highly trained athletes, it is often critically important to determine whether LV hypertrophy is an expression of a physiologic and benign adaptation to training or rather a pathologic process with risk for sudden death, such as HCM. Because there is presently no single testing approach available that will definitively resolve this question in all such athletes, several non-invasive strategies have evolved, including that reported here by Anastasakis et al ${ }^{16}$ which offers a measure of resolution to this compelling diagnostic dilemma, so important to the highly visible problem of sudden death in young competitive athletes.

I have no competing interests to declare.

\section{REFERENCES}

1 Maron BJ. Sudden death in young athletes. N Engl J Med 2000;349:1064-75.

2 Maron BJ. Sudden death in young athletes: lessons from the Hank Gathers affair. N Engl J Med 1993;329:55-7.

3 Corrado D, Pelliccia A, Biørnstad HH, et al. Cardiovascular preparticipation screening of young competitive athletes for prevention of sudden death: proposal for a common European protocol. Eur Heart J 2005;26:516-24.

4 Corrado D, Basso C, Schiavon M, et al. Screening for hypertrophic cardiomyopathy in young athletes. N Engl J Med 1998;339:364-9.

5 Maron BJ. How should we screen competitive athletes for cardiovascular disease? [editorial]. Eur Heart J 2005;26:428-30.

6 Maron BJ, Shen W-K, Link MS, et al. Efficacy of implantable cardioverterdefibrillators for the prevention of sudden death in patients with hypertrophic cardiomyopathy. N Engl J Med 2000;342:365-73. 
7 Corrado D, Leoni L, Link MS, et al. Implantable cardioverter-defibrillator therapy for prevention of sudden death in patients with arrhythmogenic right ventricular cardiomyopathy/dysplasia. Circulation 2003;108:3084-91.

8 Maron BJ, Zipes DP. 36th Bethesda Conference: eligibility recommendations for competitive athletes with cardiovascular abnormalities. J Am Coll Cardiol 2005:45:1312-75.

9 Maron BJ, Shirani J, Poliac LC, et al. Sudden death in young competitive athletes: Clinical, demographic and pathological profiles. JAMA 1996;276:199-204.

10 Maron BJ, Carney KP, Lever HM, et al. Relationship of race to sudden cardiac death in competitive athletes with hypertrophic cardiomyopathy. J Am Coll Cardiol 2003;41:974-80.

11 Maron BJ, Pelliccia A, Spirito P. Cardiac disease in young trained athletes. Insights into methods for distinguishing athlete's heart from structural heart disease, with particular emphasis on hypertrophic cardiomyopathy. Circulation 1995;91:1596-601.
12 Maron BJ, Seidman JG, Seidman CE. Proposal for contemporary screening strategies in families with hypertrophic cardiomyopathy. J Am Coll Cardiol 2004;44:2125-32

13 Pelliccia A, Maron BJ, Culasso F, et al. Athlete's heart in women: echocardiographic characterization of highly trained elite female athletes. JAMA 1996:276:211-15.

14 Sarto P, Merlo L, Noventa D, et al. Electrocardiographic changes associated with training and discontinuation of training in an athlete with hypertrophic cardiomyopathy. Am J Cardiol 2004;93:518-19.

15 Pelliccia A, Maron BJ, Spataro A, et al. Absence of left ventricular hypertrophy in athletes engaged in intense power training. Am J Cardiol 1993;72:1048-54

16 Anastasakis A, Kotsiopoulou C, Rigopoulos A, et al. Similarities in the profile of cardiopulmonary exercise testing between patients with hypertrophic cardiomyopathy and strength athletes. Heart 2005:91:1477-8.

\section{IMAGES IN CARDIOLOGY}

\section{Piffalls in the echocardiographic diagnosis of isolated non-compaction of the ventricular} myocardium

A 66 year old man presented with a one year history of atypical chest pain. The physical examination was normal, the ECG showed sinus rhythm and left anterior fascicular block, and the exercise test was normal. Two echocardiograms were performed, demonstrating an apical obliteration, which led to suspicion of endomyocardial fibrosis or apical tumour. Magnetic resonance imaging (MRI) was undertaken, which showed a typical pattern of an apical inner zone of non-compacted myocardium (NCM) distinguished from the thin outer zones of compacted myocardium (panels A, B). Late enhancement with gadolinium was also normal. The echocardiogram with second harmonic imaging was repeated (panel C) with a more detailed evaluation of the left ventricular apex, revealing trabeculations with intertrabecular recesses perfused from the left ventricular cavity (panel D). The left ventricle presented with mild dilation with mild globally depressed systolic function, and mild central mitral regurgitation. The pulsed wave tissue Doppler of septal mitral annulus showed an $\mathrm{E}^{\prime}$ peak velocity of $5.9 \mathrm{~cm} / \mathrm{s}$, characterising impaired relaxation, and $\mathrm{E} / \mathrm{E}^{\prime}$ of 6.6 , demonstrating a normal left ventricular end diastolic pressure. Screening showed one sister with the same echocardiographic pattern and one brother with two parallel false tendons extended between the interventricular septum and lateral wall.

Although echocardiography is the most frequently used first line diagnostic tool for NCM, sometimes the diagnosis is uncertain. Thus, in patients with a ventricular mass, low scale colour flow mapping demonstrates blood flow through deep recesses in continuity with the ventricular cavity. In addition, other imaging modality such as MRI, transoesophageal echocardiography, or contrast enhanced echocardiography may be performed to confirm the diagnosis.

V M C Salemi

A Q Araujo

E Arteaga

C Mady

verasalemi@uol.com.br
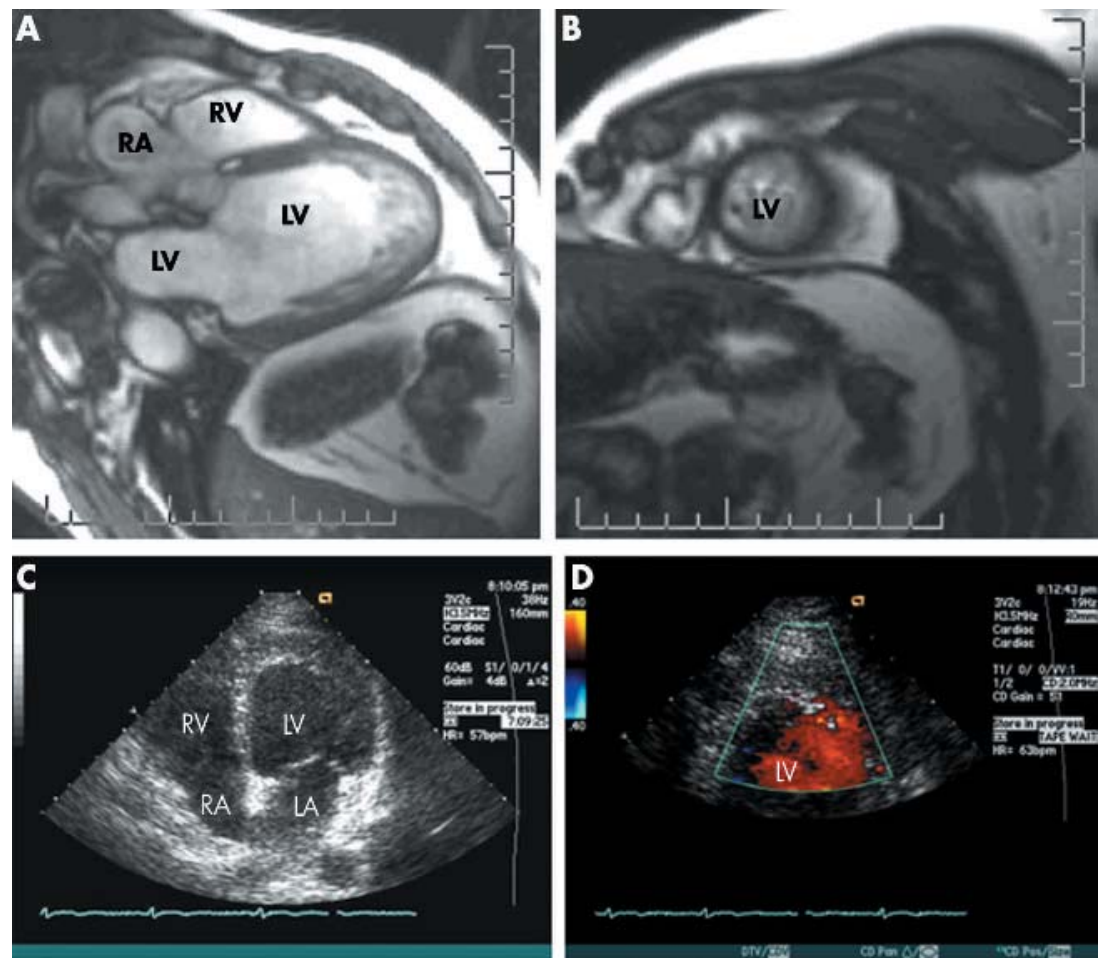